\title{
Complications, Reasons for Reoperation, and 5-Year Prosthesis Survival Compared Between the Cemented and Cementless Oxford Unicompartmental Knee Arthroplasty Fixation Techniques at Thailand's Largest National Tertiary Referral Center
}

Jirayu Paugchawee

Faculty of medicine Siriraj hospital, Mahidol university, Thailand

Chaturong Pornrattanamaneewong ( $\nabla$ toonchaturong@gmail.com )

Faculty of medicine Siriraj hospital, Mahidol university, Thailand

Pakpoom Ruangsomboon

Faculty of medicine Siriraj hospital, Mahidol university, Thailand

Rapeepat Narkbunnam

Faculty of medicine Siriraj hospital, Mahidol university, Thailand

Keerati Chareancholvanich

Faculty of medicine Siriraj hospital, Mahidol university, Thailand

Research Article

Keywords: Complications, reasons for reoperation, 5-year implant survival, Oxford unicompartmental knee arthroplasty (OUKA), Thailand

Posted Date: June 16th, 2021

DOI: https://doi.org/10.21203/rs.3.rs-583930/v1

License: (c) (1) This work is licensed under a Creative Commons Attribution 4.0 International License. Read Full License 


\section{Abstract}

Background: Oxford unicompartmental knee arthroplasty (OUKA) yields favorable outcomes in patients with medial compartmental knee osteoarthritis; however, it remains unknown whether cemented or cementless OUKA fixation delivers better outcomes in Asian population. Accordingly, this study aimed to investigate the complications, reasons for reoperation, and 5-year prosthesis survival compared between cemented and cementless OUKA in Thai patients.

Methods: A total of 466 cemented and 36 cementless OUKA that were performed during 2011-2015 with a minimum follow-up of five years were included. With reoperation for any reason as the endpoint, Kaplan-Meier analysis was performed to compare 5-year implant survival between groups. Complications, reasons for reoperation, and 90-day morbidity and mortality were compared between groups. Cox proportional hazards model was used to identify independent predictors of implant survival.

Results: There was no significant difference in 5-year implant survival between the cemented and cementless groups $(96.4 \%$ vs. $94.4 \%, p=0.375)$. The mean implant survival time was $113.0 \pm 0.8$ and $70.8 \pm 1.9$ months in the cemented and cementless groups, respectively $(p=0.383)$. The most common reason for reoperation was bearing dislocation, and only one patient had 90 -day morbidity. There was no significant difference between groups for complications or reasons for reoperation. No independent predictors of implant survival were identified in multivariate analysis.

Conclusions: OUKA was shown to be a safe and durable reconstructive procedure in Thai patients with medial compartmental knee osteoarthritis. There was no significant difference in implant survival between the cemented and cementless groups during the 5-year follow-up, and no independent predictors of implant survival were identified.

Trial registration: Thai Clinical Trials Registry, TCTR20200427004. Registered 27 April 2020 Retrospectively registered.

\section{Background}

Unicompartmental knee arthroplasty is an effective treatment for medial compartmental osteoarthritis knee patients who have failed conservative treatment (1-3). Oxford UKA (OUKA) is a fully congruent mobile bearing prosthesis with spherical femoral and flat tibial components. After design modifications, the OUKA phase 3 design was introduced in 1998, and it was reported to deliver good outcomes and longevity $(4,5)$.

There are two types of fixation that can be employed to implant the OUKA prosthesis. Cemented fixation was initially the only option for implantation. Later - to achieve biologic fixation, a cementless version with a porous coating of titanium and hydroxyapatite, and an additional peg in the femoral component was introduced in 2004. The cementless version can facilitate avoidance of cementing error, and it can reduce radiographic radiolucent lines that may be misinterpreted as a sign of loosening $(6,7)$. 
Kerens, et al. (8) reported no significant difference in implant survival between the cemented $(84 \%$ at 54 months) and cementless OUKA ( $90 \%$ at 34 months) fixation techniques. From South Africa, Campi, et al. (9) also reported similar 5-year survivorship between the two OUKA fixation methods. When considering the results from the more extensive and longer-term studies, Mohammad, et al. (5) collected data from the National Joint Registry for England, Wales, Northern Ireland, and the Isle of Man (NJR). They found the 10 -year cumulative survival of cemented OUKA $(90 \%, 95 \% \mathrm{Cl}: 88-92 \%)$ to be significantly lower than that of cementless OUKA (93\%, 95\% Cl: 90-96\%). Gupta, et al. (3) performed a 19-year analysis of data from the New Zealand Joint Registry (NZJR), and they found that cemented OUKA had a significantly higher rate of revision over time compared to cementless OUKA, and the risk of revision was $>1.8$-fold (hazard ratio: 1.82, 95\% Cl: 1.27-2.60). However, all of the studies mentioned above were conducted in Western populations, and no comparative study has yet been reported from Asian countries.

Several studies have reported differences in knee morphology between Caucasians and Asians (10-12). These differences may be due to differences in lifestyle, including high knee flexion and floor activity in Asian cultures, and these factors may influence the rates of implant failure and survival. The aim of this study was to investigate the complications, reasons for reoperation, and 5-year implant survival compared between the cemented and cementless OUKA fixation techniques in Thai medial compartmental knee osteoarthritis patients.

\section{Methods}

This retrospective chart review identified 572 patients who underwent OUKA for treatment of medial compartmental osteoarthritic knee during 2011 to 2015 at our institute. Seventy knees were subsequently excluded due to incomplete data or follow-up time $<5$ years. The remaining 502 knees were categorized into the cemented or cementless fixation groups. Five experienced arthroplasty surgeons performed all procedures. The protocol for the present study received approval from our institutional review board, and written informed consent was waived due to our study's retrospective design.

Baseline characteristics, including age, gender, weight, height, body mass index (BMI), and American Association of Anesthesiologists (ASA) classification, were collected and recorded. For intraoperative parameters, tourniquet time and implant component sizes were collected. Postoperative complications, 90-day morbidity (including infection, fracture, venous thromboembolism, cerebrovascular, and cardiovascular events) and 90-day mortality were also recorded and analyzed. During the follow-up period, the reoperation rate (for any reason) and reasons for reoperation were also collected and included in our analysis.

\section{Statistical analysis}

Data were analyzed using SPSS Statistics version 18.0. (SPSS Inc., Chicago, IL, USA). Continuous data were presented as mean \pm standard deviation, and categorical data were presented as number and percentage. Kaplan-Meier survival analysis was used to compare OUKA implant survival between the 
cemented and cementless fixation groups. The starting point was the date of operation, and the endpoint was the date of reoperation for any reason. Log-rank test was used to compare the survival curves between groups. Univariate analysis was performed using Student's $t$-test for continuous data, and using chi-square or Fisher's exact test for categorical data. Cox proportional hazards model was used to identify independent predictors of OUKA implant survival. A $p$-value less than 0.05 was considered to be statistically significant.

\section{Results}

Patient demographic data, clinical characteristics, and intraoperative parameters are shown in Table 1. The cemented group was significantly older than the cementless group (65.4 \pm 8.2 vs. 56.6 \pm 3.8 years, $p<0.001)$. Significant differences in ASA classification and femoral component sizing were also observed between groups ( $p=0.018$ and $p=0.036$, respectively). The cemented group had significantly longer tourniquet time than the cementless group $(66.0 \pm 20.7$ vs. $58.0 \pm 11.5$ minutes, $p=0.001)$.

Regarding in-hospital complications, 90-day morbidity, and 90-day mortality, one patient in the cemented group had an acute-on-chronic pulmonary embolism on the first postoperative day with subsequent medical treatment success. There were no in-hospital complications or 90-day mortality in our series. The

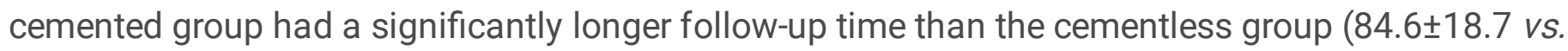
$64.4 \pm 10.5$ months, respectively; $p<0.001$ ) (Table 2).

Regarding the primary outcome, the 5-year OUKA implant survival rate in the cemented and cementless groups was $96.4 \%$ and $94.4 \%$, respectively. There was no significant difference between groups $(p=0.375)$. The overall reoperation rate of OUKA in this series was $3.6 \%$, and the overall mean survival time of OUKA in our series was $112.8 \pm 0.7$ months. Kaplan-Meier survival analysis compared between the two fixation groups is shown in Figure 1. There was no significant difference in the mean implant survival time between the cemented and cementless groups (113.0 \pm 0.8 months, $95 \%$ Cl: $111.5-114.5$ vs. $70.8 \pm 1.9$ months, $95 \% \mathrm{Cl}$ : $67.2-74.4$ months, respectively; $p=0.383)$. There was also no significant difference in the reasons for reoperation between groups (Table 2). Details specific to cases that required reoperation are described in Table 3.

The type of prosthesis and statistically significant factors from univariate analysis (including age, ASA, and femoral component sizing) were entered into Cox proportional hazards model to identify independent predictors of OUKA implant survival. The results of that analysis revealed no independent associations (all $p>0.05$ ).

\section{Discussion}

To our knowledge, this is the first study to compare implant survival between cemented and cementless OUKA in Asian patients. The most important finding of this study is that we observed no significant difference in 5-year implant survival between the two fixation methods. This result is similar to the 
findings of studies reported from the West. Akan, et al. (13) performed a retrospective study that found no significant difference in the revision rate between cemented and cementless OUKA ( $7.1 \%$ vs. $4.9 \%$.

$p=0.155)$ at a mean follow-up time of 42 and 30 months, respectively. The multicenter retrospective study of Kerens, et al. revealed no significant difference in the implant survival rates between cemented ( $84 \%$ at 54 months) and cementless OUKA ( $90 \%$ at 34 months). Pandit, et al. (14) conducted a randomized controlled trial of 33 cemented and 30 cemented OUKA. At the 5-year follow-up, no revision cases were reported in their study. Stempin, et al. collected data from a prospective cohort study with an average follow-up time of 7 years and found no significant difference in the revision rate between cemented (3.4\%) and cementless OUKA (2.0\%). In South Africa, Campi, et al. (9) performed a survival analysis in a prospective cohort of 522 cemented and 598 cementless OUKA. Their results showed no significant difference in 5-year implant survival between cemented and cementless OUKA (95.1\% vs. 95.8\%, $p=0.97)$.

However, contradictory results were reported from larger or longer-term studies. Knifsund, et al. (15) collected data from the Finnish Arthroplasty Register, and they found the 5-year survival of cemented OUKA to be significantly shorter than that of cementless OUKA $(88.9 \%$ vs. $92.3 \%, p<0.05)$. A large propensity-matched NJR-based study also showed the risk of revision in cementless OUKA to be $24 \%$ less than that of cemented OUKA within 10 years (5). Additionally, a recent meta-analysis of 901 patients revealed cementless OUKA to be associated with a lower revision rate after follow-up for at least two years (odds ratio: $1.83,95 \%$ confidence interval: 0.90-3.73).

Considering the different types of OUKA failure in our study, the most common reason for reoperation in cemented OUKA was bearing dislocation. In cementless OUKA, the most common reasons were osteoarthritis progression and infection. Our study's reoperation rate for bearing dislocation was higher than the rates from Western registry-based data $(5,15)$. A meta-analysis by Ro, et al. $(16)$ found the mean reoperation rate per 100 observed component years for bearing dislocation after OUKA to be higher in Asians $(0.53,95 \% \mathrm{Cl}: 0.41-0.64)$ than in Westerners $(0.14 ; 95 \% \mathrm{Cl}: 0.12-0.17)$. There are factors that may explain this problem. Hyperflexion knee or floor activities are commonly performed in Asian populations, and these activities resulted in anterior tibial subluxation and strained anterior cruciate ligament that increased the risk of bearing dislocation (17). Another possible explanation is that repetitive hyperflexion activity might cause impingement between the polyethene bearing and the remaining osteophytes or meniscus $(12,17)$. Furthermore, a relatively smaller tibia in Asians compared to Westerners result in tibial component size mismatch that could lead to mediolateral overhang of the tibial component. This overhang creates more space for bearing motion and might cause an imbalance in soft tissue tension. This hypothesis may also increase the chance of bearing dislocation.

The results of our study also demonstrated OUKA to be a safe procedure for treating medial compartmental knee osteoarthritis in Asians relative to complications, morbidity, and mortality. We had only one patient with 90-day morbidity in our series, with no complications and no mortality. This is similar to the results from a study in 2,316 OUKA from the National Surgical Quality Improvement Program that reported an overall complication and 90-day morbidity rate of $3.2 \%$ with no mortality (18). 


\section{Limitations}

This study also has some mentionable limitations. First, our study had a comparatively small number of cases enrolled in the cementless group because the cementless prosthesis was introduced in Thailand in 2014. It is possible that the small number of cementless cases could have limited the statistical power of our study to identify independent predictors of OUKA implant survival. Gupta, et al. (3) found that cemented OUKA had superior implant survival in women aged $<65$ years, and in men aged 55 to 74 . They suggested age and gender to be important factors to consider when determining the OUKA fixation technique. Second, our study reported only short- to mid-term implant survival, and study of longer-term outcomes is needed. Third, all procedures were performed by medium- to high-volume surgeons. As such, different outcomes might be observed in low-volume surgeons. From the NJR database, the 10-year survival rate for cemented and cementless OUKA was $86.8 \%$ and $81.8 \%$ in low-volume surgeons ( $<10$ cases/year); $94.3 \%$ and $92.5 \%$ for medium-volume surgeons ( 10 to $<30$ cases/years); and, $97.5 \%$ and $94.2 \%$ for high-volume surgeons ( $\geq 30$ cases/year), all respectively (19). Fourth and last, because of this study's retrospective design, we were not able to evaluate functional or patient-reported outcomes. Implant survivorship was the only outcome that could be confidently assessed. However, many of these outcomes have already been studied in previous trials.

\section{Conclusion}

OUKA was shown to be a safe and durable reconstructive procedure in Thai patients with medial compartmental knee osteoarthritis. There was no significant difference in implant survival between the cemented and cementless groups during the 5-year follow-up, and no independent predictors of implant survival were identified. The most common reason for reoperation was bearing dislocation.

\section{Abbreviations}

OUKA: Oxford unicompartmental knee arthroplasty; NJR: National Joint Registry for England, Wales, Northern Ireland, and the Isle of Man; NZJR: New Zealand Joint Registry; BMI: body mass index; ASA: American Association of Anesthesiologists

\section{Declarations}

\section{Ethics approval and consent to participate}

This study was approved by Siriraj institutional review board (879/2562 (IRB3)). The written informed consent was waived due to the retrospective design.

\section{Consent for publication}

Not applicable 


\section{Availability of data and materials}

The data that support the results of this study are available from the corresponding author upon reasonable request.

\section{Competing interests}

All authors declare no personal or professional conflicts of interest, and no financial support from the companies that produce and/or distribute the drugs, devices, or materials described in this report.

\section{Funding}

This was an unfunded study.

\section{Authors 'contributions}

JP carried out the data collection and drafted the manuscript. PR, RP and KC participated in the data collection and interpreted the data. CP conceived the study design, interpreted the data, checked the statistical analysis and drafted the manuscript. All authors read and approved the final manuscript.

\section{Acknowledgements}

The authors gratefully acknowledge Miss Nichakorn Khomawut for assistance with statistical analysis.

\section{References}

1. Murray DW, Goodfellow JW, O'Connor JJ. The Oxford medial unicompartmental arthroplasty: a tenyear survival study. J Bone Joint Surg Br. 1998 Nov;80(6):983-9.

2. Lisowski LA, van den Bekerom MPJ, Pilot P, van Dijk CN, Lisowski AE. Oxford Phase 3 unicompartmental knee arthroplasty: medium-term results of a minimally invasive surgical procedure. Knee Surg Sports Traumatol Arthrosc Off J ESSKA. 2011 Feb;19(2):277-84.

3. Gupta V, Kejriwal R, Frampton C. Revision Following Cemented and Uncemented Oxford-III Primary Medial Unicompartmental Knee Replacements: A 19-Year Analysis from the New Zealand Joint Registry. J Bone Joint Surg Am. 2020 Oct 21;102(20):1777-83.

4. Walker T, Hetto P, Bruckner T, Gotterbarm T, Merle C, Panzram B, et al. Minimally invasive Oxford unicompartmental knee arthroplasty ensures excellent functional outcome and high survivorship in the long term. Knee Surg Sports Traumatol Arthrosc Off J ESSKA. 2019 May;27(5):1658-64. 
5. Mohammad HR, Matharu GS, Judge A, Murray DW. Comparison of the 10-year outcomes of cemented and cementless unicompartmental knee replacements: data from the National Joint Registry for England, Wales, Northern Ireland and the Isle of Man. Acta Orthop. 2020;91(1):76-81.

6. Pandit HG, Campi S, Hamilton TW, Dada OD, Pollalis S, Jenkins C, et al. Five-year experience of cementless Oxford unicompartmental knee replacement. Knee Surg Sports Traumatol Arthrosc Off J ESSKA. 2017 Mar;25(3):694-702.

7. Panzram B, Bertlich I, Reiner T, Walker T, Hagmann S, Weber M-A, et al. Results after Cementless Medial Oxford Unicompartmental Knee Replacement - Incidence of Radiolucent Lines. PloS One. 2017;12(1):e0170324.

8. Kerens B, Schotanus MGM, Boonen B, Boog P, Emans PJ, Lacroix H, et al. Cementless versus cemented Oxford unicompartmental knee arthroplasty: early results of a non-designer user group. Knee Surg Sports Traumatol Arthrosc Off J ESSKA. 2017 Mar;25(3):703-9.

9. Campi S, Pandit HG, Oosthuizen CR. The Oxford Medial Unicompartmental Knee Arthroplasty: The South African Experience. J Arthroplasty. 2018;33(6):1727-31.

10. Urabe K, Mahoney OM, Mabuchi K, Itoman M. Morphologic differences of the distal femur between Caucasian and Japanese women. J Orthop Surg Hong Kong. 2008 Dec;16(3):312-5.

11. Shao H, Chen C, Scholl D, Faizan A, Chen AF. Tibial shaft anatomy differs between Caucasians and East Asian individuals. Knee Surg Sports Traumatol Arthrosc Off J ESSKA. 2018 Sep;26(9):2758-65.

12. Yoshida S, Aoyagi K, Felson DT, Aliabadi P, Shindo H, Takemoto T-I. Comparison of the prevalence of radiographic osteoarthritis of the knee and hand between Japan and the United States. J Rheumatol. 2002 Jul;29(7):1454-8.

13. Akan B, Karaguven D, Guclu B, Yildirim T, Kaya A, Armangil M, et al. Cemented versus Uncemented Oxford Unicompartmental Knee Arthroplasty: Is There a Difference? Adv Orthop. 2013;2013:245915.

14. Pandit $H$, Liddle AD, Kendrick BJL, Jenkins $C$, Price AJ, Gill HS, et al. Improved fixation in cementless unicompartmental knee replacement: five-year results of a randomized controlled trial. J Bone Joint Surg Am. 2013 Aug 7;95(15):1365-72.

15. Knifsund J, Reito A, Haapakoski J, Niinimäki T, Eskelinen A, Leskinen J, et al. Short-term survival of cementless Oxford unicondylar knee arthroplasty based on the Finnish Arthroplasty Register. The Knee. 2019 Jun;26(3):768-73.

16. Ro K-H, Heo J-W, Lee D-H. Bearing Dislocation and Progression of Osteoarthritis After Mobile-bearing Unicompartmental Knee Arthroplasty Vary Between Asian and Western Patients: A Meta-analysis. Clin Orthop. 2018 May;476(5):946-60.

17. Kim JM, Moon MS. Squatting following total knee arthroplasty. Clin Orthop. 1995 Apr;(313):177-86.

18. Haughom BD, Schairer WW, Hellman MD, Nwachukwu BU, Levine BR. An Analysis of Risk Factors for Short-Term Complication Rates and Increased Length of Stay Following Unicompartmental Knee Arthroplasty. HSS J Musculoskelet J Hosp Spec Surg. 2015 Jul;11(2):112-6.

19. Mohammad HR, Matharu GS, Judge A, Murray DW. The Effect of Surgeon Caseload on the Relative Revision Rate of Cemented and Cementless Unicompartmental Knee Replacements: An Analysis 
from the National Joint Registry for England, Wales, Northern Ireland and the Isle of Man. J Bone Joint Surg Am. 2020 Apr 15;102(8):644-53.

\section{Tables}


Table 1

Patient demographic data, clinical characteristics, and intraoperative parameters compared between the cemented and cementless OUKA groups

\begin{tabular}{|c|c|c|c|}
\hline Variables & $\begin{array}{l}\text { Cemented group } \\
(n=466)\end{array}$ & $\begin{array}{l}\text { Cementless group } \\
(n=36)\end{array}$ & p-value \\
\hline Age (years), mean $\pm S D$ & $65.4 \pm 8.2$ & $56.6 \pm 3.8$ & $<0.001$ \\
\hline Female gender, $\mathrm{n}(\%)$ & 397 (85.2\%) & $32(88.9 \%)$ & 0.544 \\
\hline Weight $(\mathrm{kg})$, mean \pm SD & $67.1 \pm 11.4$ & $69.8 \pm 13.8$ & 0.179 \\
\hline Height $(\mathrm{cm})$, mean \pm SD & $156.1 \pm 6.9$ & $155.7 \pm 5.5$ & 0.736 \\
\hline $\mathrm{BMI}\left(\mathrm{kg} / \mathrm{m}^{2}\right)$, mean $\pm \mathrm{SD}$ & $27.5 \pm 4.3$ & $28.8 \pm 5.6$ & 0.188 \\
\hline Right side, n (\%) & $232(49.8 \%)$ & $22(61.1 \%)$ & 0.190 \\
\hline ASA classification, n (\%) & & & 0.018 \\
\hline I & $52(11.2 \%)$ & $7(19.4 \%)$ & \\
\hline II & $357(76.6 \%)$ & $20(55.6 \%)$ & \\
\hline III & $57(12.2 \%)$ & $9(25.0 \%)$ & \\
\hline Tourniquet time $(\min )$, mean $\pm S D$ & $66.0 \pm 20.7$ & $58.0 \pm 11.5$ & 0.001 \\
\hline Femoral component sizing, n (\%) & & & 0.036 \\
\hline Extra small & $113(24.2 \%)$ & $2(5.6 \%)$ & \\
\hline Small & $250(53.7 \%)$ & $27(75.0 \%)$ & \\
\hline Medium & $86(18.5 \%)$ & $5(13.8 \%)$ & \\
\hline Large & $17(3.6 \%)$ & $2(5.6 \%)$ & \\
\hline Tibial component sizing, n (\%) & & & 0.295 \\
\hline AA & $136(29.2 \%)$ & $10(27.8 \%)$ & \\
\hline A & $166(35.6 \%)$ & $19(52.8 \%)$ & \\
\hline B & $83(17.8 \%)$ & $5(13.8 \%)$ & \\
\hline C & $62(13.3 \%)$ & $1(2.8 \%)$ & \\
\hline D & 18 (3.9\%) & $1(2.8 \%)$ & \\
\hline$E$ & $1(0.2 \%)$ & $0(0.0 \%)$ & \\
\hline
\end{tabular}




\begin{tabular}{|llll|}
\hline Variables & $\begin{array}{l}\text { Cemented group } \\
(\mathbf{n = 4 6 6 )}\end{array}$ & $\begin{array}{l}\text { Cementless group } \\
(\mathbf{n = 3 6 )}\end{array}$ & p-value \\
\hline Polyethylene thickness, $\mathrm{n}(\%)$ & & & \\
\hline $3 \mathrm{~mm}$ & $198(42.5 \%)$ & $14(38.9 \%)$ & \\
\hline $4 \mathrm{~mm}$ & $165(35.4 \%)$ & $13(36.1 \%)$ & \\
\hline $5 \mathrm{~mm}$ & $71(15.2 \%)$ & $6(16.6 \%)$ & \\
\hline $6 \mathrm{~mm}$ & $20(4.3 \%)$ & $2(5.6 \%)$ & \\
\hline $7 \mathrm{~mm}$ & $11(2.4 \%)$ & $1(2.8 \%)$ & $0(0.0 \%)$ \\
\hline $8 \mathrm{~mm}$ & $1(0.2 \%)$ & & \\
\hline $\begin{array}{l}\text { A p-value< } 0.05 \text { indicates statistical significance } \\
\text { Abbreviations: OUKA, Oxford unicompartmental knee arthroplasty; SD, standard deviation; BMI, body } \\
\text { mass index; ASA, American Association of Anesthesiologists }\end{array}$ & \\
\hline
\end{tabular}


Table 2

Postoperative outcomes compared between the cemented and cementless OUKA groups

\begin{tabular}{|c|c|c|c|}
\hline Outcomes & $\begin{array}{l}\text { Cemented group } \\
(n=466)\end{array}$ & $\begin{array}{l}\text { Cementless group } \\
(n=36)\end{array}$ & p \\
\hline In-hospital complications, n (\%) & $0(0.0 \%)$ & $0(0.0 \%)$ & NA \\
\hline 90-day morbidity (\%) & $1(0.2 \%)$ & $0(0.0 \%)$ & 1.000 \\
\hline 90-day mortality (\%) & $0(0.0 \%)$ & $0(0.0 \%)$ & NA \\
\hline \multicolumn{4}{|l|}{ Follow-up period } \\
\hline Follow-up time (months), mean \pm SD & $84.6 \pm 18.7$ & $64.4 \pm 10.5$ & $<0.001$ \\
\hline Reoperation, n (\%) & $16(3.4 \%)$ & $2(5.6 \%)$ & 0.375 \\
\hline \multicolumn{2}{|l|}{ Reasons for reoperation, $\mathrm{n}(\%)$} & & 0.723 \\
\hline Aseptic loosening & $3(18.8 \%)$ & $0(0.0 \%)$ & \\
\hline Bearing dislocation & $5(31.3 \%)$ & $0(0.0 \%)$ & \\
\hline Periprosthetic joint infection & $3(18.8 \%)$ & $1(50.0 \%)$ & \\
\hline Lateral compartmental OA & $2(12.5 \%)$ & $1(50 \%)$ & \\
\hline Patellofemoral OA & $1(6.3 \%)$ & $0(0.0 \%)$ & \\
\hline Unexplained pain & $1(6.3 \%)$ & $0(0.0 \%)$ & \\
\hline Periprosthetic fracture & $1(6.3 \%)$ & $0(0.0 \%)$ & \\
\hline Prosthesis survival time (months), mean \pm SD & $113.0 \pm 0.8$ & $70.8 \pm 1.9$ & 0.383 \\
\hline \multicolumn{4}{|c|}{$\begin{array}{l}\text { A p-value < } 0.05 \text { indicates statistical significance } \\
\text { Abbreviations: OUKA, Oxford unicompartmental knee arthroplasty; SD, standard deviation; OA, } \\
\text { osteoarthritis }\end{array}$} \\
\hline
\end{tabular}


Table 3

Reasons for reoperation and management in the cemented and cementless OUKA groups

\begin{tabular}{|c|c|c|c|}
\hline $\begin{array}{l}\text { Months since } \\
\text { operation }\end{array}$ & $\begin{array}{l}\text { Type of } \\
\text { prosthesis }\end{array}$ & Reasons for reoperation & Management \\
\hline 5 & Cemented & Periprosthetic joint infection & $\begin{array}{l}\text { Arthrotomy, debridement, and } \\
\text { bearing exchange }\end{array}$ \\
\hline 6 & Cementless & Periprosthetic joint infection & $\begin{array}{l}\text { Arthrotomy, debridement, and } \\
\text { bearing exchange }\end{array}$ \\
\hline 9 & Cemented & Periprosthetic joint infection & $\begin{array}{l}\text { Arthrotomy, debridement, and } \\
\text { bearing exchange }\end{array}$ \\
\hline 10 & Cemented & $\begin{array}{l}\text { Aseptic loosening of tibial } \\
\text { component }\end{array}$ & Revision to TKA \\
\hline 15 & Cemented & Bearing dislocation & Bearing upsize \\
\hline 16 & Cemented & Patellofemoral osteoarthritis & Patellofemoral joint arthroplasty \\
\hline 18 & Cemented & $\begin{array}{l}\text { Aseptic loosening of tibial } \\
\text { component }\end{array}$ & Revision to TKA \\
\hline 18 & Cemented & $\begin{array}{l}\text { Aseptic loosening of femoral } \\
\text { component }\end{array}$ & Revision to TKA \\
\hline 20 & Cemented & Bearing dislocation & Revision to TKA \\
\hline 23 & Cemented & Bearing dislocation & Bearing upsize \\
\hline 24 & Cemented & Bearing dislocation & Revision to TKA \\
\hline 30 & Cemented & Bearing dislocation & Revision to TKA \\
\hline 32 & Cemented & Unexplained pain & Revision to TKA \\
\hline 50 & Cemented & Periprosthetic joint infection & $\begin{array}{l}\text { Arthrotomy, debridement, and } \\
\text { bearing exchange }\end{array}$ \\
\hline 61 & Cementless & $\begin{array}{l}\text { Lateral compartmental } \\
\text { osteoarthritis }\end{array}$ & Revision to TKA \\
\hline 65 & Cemented & $\begin{array}{l}\text { Lateral compartmental } \\
\text { osteoarthritis }\end{array}$ & Revision to TKA \\
\hline 83 & Cemented & Medial tibial plateau fracture & ORIF with plate and screws \\
\hline 92 & Cemented & $\begin{array}{l}\text { Lateral compartmental } \\
\text { osteoarthritis }\end{array}$ & Revision to TKA \\
\hline
\end{tabular}


Figures

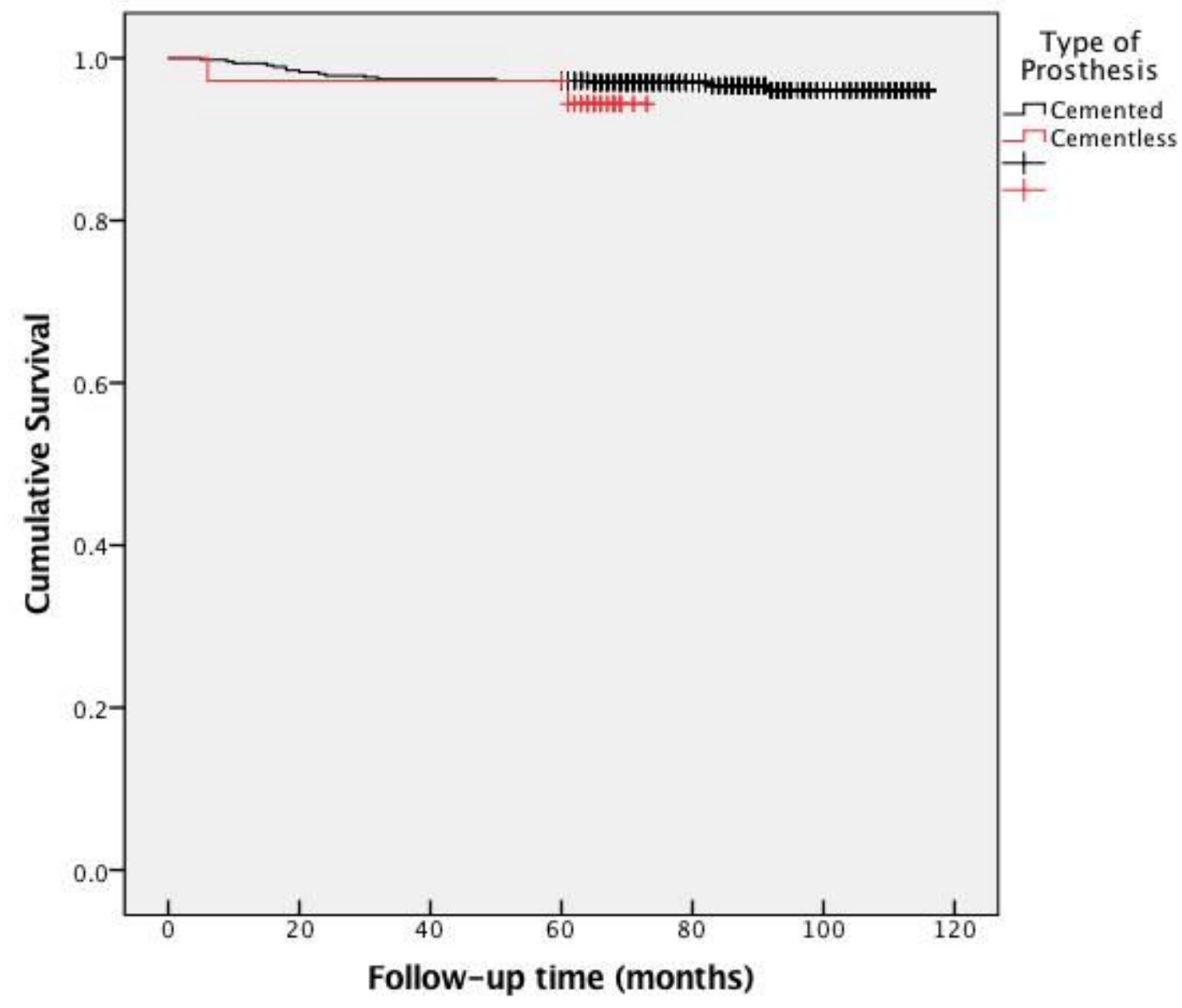

\section{Figure 1}

Kaplan-Meier analysis of Oxford unicompartmental knee arthroplasty (OUKA) cumulative survival during the follow-up period compared between the cemented and cementless OUKA groups 